\title{
Channel characterization for air-to-ground free-space optical communication links
}

\author{
Kevin Shortt, Dirk Giggenbach, Ramon Mata-Calvo, Florian Moll, Christian Fuchs, Christopher \\ Schmidt, Joachim Horwath, Jack Yeh, Vevek Selvaraj, Ranjoy Banerjee \\ Optical Communications Group, Institute for Communications and Navigation, German Aerospace Center (DLR), \\ 82234 Weßling, Germany
}

\begin{abstract}
The next five to ten years will see more and more free-space optical communication systems being put into practical use as technologies and techniques continue to mature, particularly in the area of mobile and satellite-to-ground communications. To meet the increasing demand of these types of systems, it is necessary to gain a deeper understanding of the various atmospheric effects at play in a free-space optical link in an effort to mitigate their impact on operational systems. In that context, the German Aerospace Center (DLR) has conducted a number of field trials between a Dornier 228 aircraft and its ground station in Oberpfaffenhofen, just south of Munich, Germany. These field trials have involved the concurrent measurement of atmospheric turbulence using three different techniques: pupil plane imaging, focus spot imaging and Shack-Hartmann wave-front sensing. To ensure the accurate synchronization of measurements between the three techniques, a concerted effort was made in the selection of computer hardware and the development of image acquisition software. Furthermore, power measurements in up- and downlink have been taken to be further correlated with the 3 primary instruments. It is envisioned that the resulting analysis of these measurements shall contribute to the implementation of new adaptive optics techniques to facilitate various air and space communication links. This paper shall describe the overall experiment design as well as some of the design decisions that led to the final experiment configuration.
\end{abstract}

Keywords: atmospheric turbulence, Shack-Hartmann, wave-front sensing, pupil plane, focal speckle pattern, mobile communications, aircraft-to-ground

\section{INTRODUCTION}

Nowadays, remote sensing platforms (e.g. aircraft, UAVs, satellites etc.) are using increasingly sophisticated sensor payloads to gather data about our world. Regardless of whether the data is time critical, such as in disaster management situations, or required to support longer term scientific goals, operators require higher bandwidth through which to transmit vast quantities of data in a timely manner. To ensure these communication links are robust enough to support the plethora of applications in which they will be used, it is necessary to establish a firm understanding of the real-world atmospheric dynamics such links will encounter. To that end, a series of flight campaigns at DLR were mounted to gain a better understanding of aircraft downlinks and to verify theoretical models with actual measurements.

Over the years, a number of experiments have been conducted to test the practical feasibility of applications such as inter-satellite links, GEO-to-ground links, LEO-to-ground links and, more recently, deep space-to-ground links. Beginning as early as November 2000, the first optical link experiments were being conducted between ESA's OPALE terminal on board the Artemis GEO satellite [1]. More recently, in the fall of 2013, MIT's Lincoln Laboratory successfully demonstrated a bi-directional link between a ground terminal and NASA's LADEE spacecraft [2]. Furthermore, research is being conducted into characterizing the atmospheric channel in Europe [3], the US [4], Japan [5] and Canada [6], to name but a few, in an effort to develop methods for mitigating phenomena such as fading events in an optical communications link. Despite these successful demonstrations and advances in research around the world, there has been a lack of experimental work examining aircraft-to-ground links. It is for this reason that the Optical Communications Group at DLR embarked upon a series of projects to advance the knowledge necessary for deploying such systems. For example, the ARGOS project examined the design criteria for an aircraft-to-ground optical communications terminal [7]. Further to this research, the VABENE project is intended to examine operational scenarios involving the real-time monitoring and management of large-scale events and disasters as they unfold [8]. Projects such

Copyright 2014 Society of Photo-Optical Instrumentation Engineers. This paper was published in SPIE Photonics West 2014 and is made available as an electronic reprint with permission of SPIE. One print or electronic copy may be made for personal use only. Systematic reproduction and distribution, duplication of any material in this paper for a fee or for commercial purposes, or modification of the content of the paper are prohibited. 
as those mentioned are all testaments to the growing need for aircraft to provide high-fidelity, real-time monitoring, necessitating the use of high bandwidth optical communication links.

One project undertaken within the wider scope of aircraft link development, known as ALTAO, was envisioned to examine alternative adaptive optics techniques to increase the fidelity of an optical communications link in the highly dynamic scenario of aircraft-to-ground links. Using the optical ground station (OGS) at DLR's Institute for Communications and Navigation in Oberpfaffenhofen, this examination involved three different methods to characterize the optical channel; focal spot analysis, pupil image analysis and Shack-Hartmann lenslet array imaging. From these different methods, atmospheric parameters such as the Fried parameter can be determined thus allowing requirements for future adaptive optic systems to be derived. In addition, as an experiment uniquely involving field trials with an aircraft, the ALTAO project provides a better understanding of aircraft downlinks through the comparison of theoretical models with actual measurement data and paves the way forward for ongoing development in this critical application area.

The remaining discussion in this paper shall provide an overview of the ALTAO experimental setup as well as a description of the flight operations. While this paper primarily focuses on the OGS instrumentation used to derive the Fried parameter, the atmospheric correlation length and scintillation index, technical highlights of the aircraft terminal are also provided. Finally, the paper concludes with a brief discussion on lessons learned during the course of the flight campaign.

\section{AIRCRAFT TERMINAL}

The aircraft terminal is the transmitter part of the laser communication system, installed in DLR's Dornier Do-228 aircraft and used during the channel characterization trial. DLR's Do-228 is a turboprop engine, short take-off and landing aircraft with a special opening in the floor of the aircraft cabin for scientific equipment.

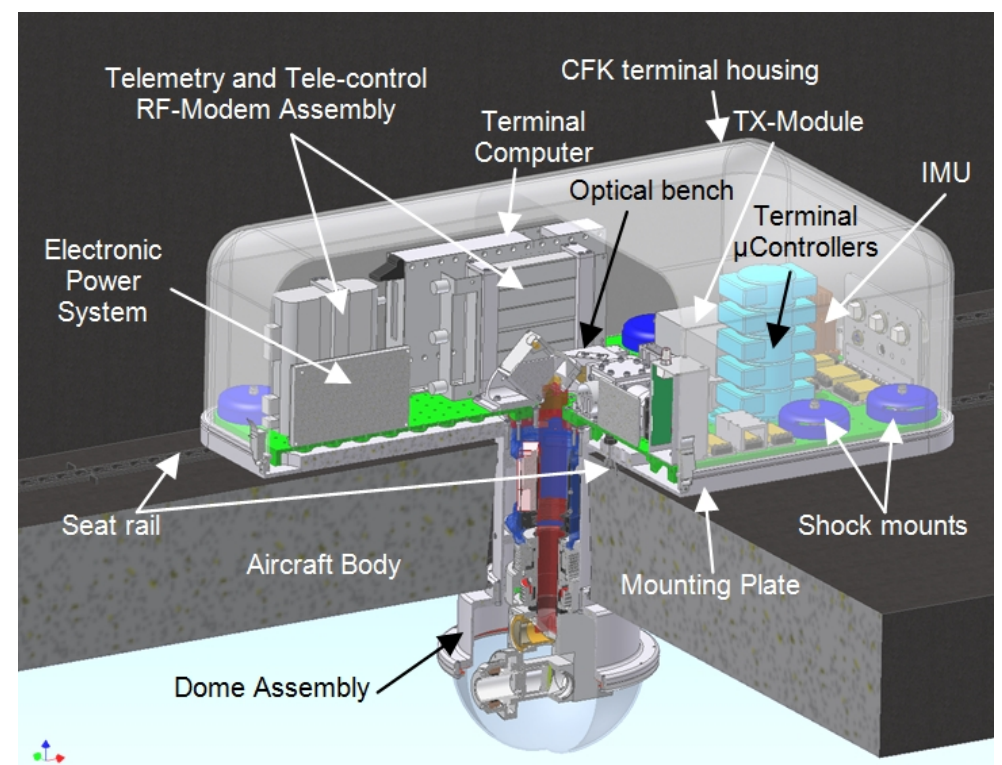

Figure 1. Detailed illustration of FELT II mounted in Do-228 [9].

Figure 1 shows a detailed illustration of the used Free-space Experimental Laser Terminal II (FELT II), which has been developed in the ARGOS and VABENE projects. Due to the turboprop engines, the Do-228 has demanding vibration characteristics for the laser terminal installed on the seat rails. Therefore, the optical bench is installed on shock mounts on the mounting plate to uncouple the optical system from the aircraft body. 
The terminal is equipped with a power system, telemetry and tele-control modem, terminal computer and terminal $\mu$ Controllers as well as the transmitter-module [9]. The transmitter module provides a maximum output power of $1 \mathrm{~W}$ at a maximum data rate of $1 \mathrm{Gbps}$.

FELT II is further equipped with a two-stage pointing system, consisting of a coarse pointing assembly (CPA) and a fine pointing assembly (FPA). The CPA is mounted in the dome assembly below the aircraft body and is capable for the coarse pointing to the ground station. For an improved tracking offset, a FPA system is used as a second stage. The FPA is mounted on the optical bench inside the aircraft cabin and consists of a tracking sensor and a fast steering mirror. Using the CPA and FPA together, the influence of turbulence and micro-vibrations can be reduced to a tracking offset typically below $200 \mu \mathrm{rad}$. Since the system has a beam divergence of $1 \mathrm{mrad}$, it more than compensates for this tracking accuracy.

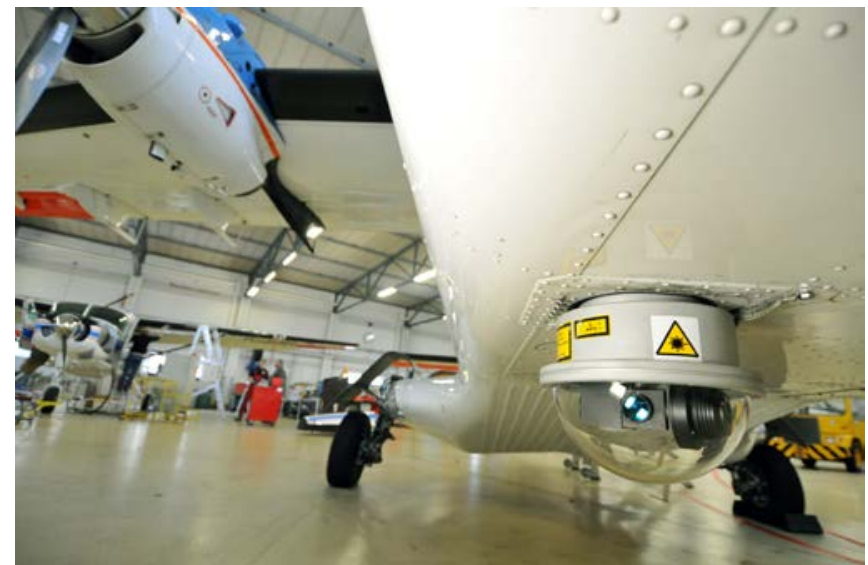

Figure 2. FELT II mounted in Do-228 with CPA in dome assembly below aircraft cabin.

Figure 2 shows the FELT II mounted in Do-228 with the dome assembly below the aircraft body. The CPA is covered by a glass dome to protect it from environmental influences in flight.

\section{OPTICAL GROUND STATION}

The Optical Ground Station in Oberpfaffenhofen (OGS-OP) operates as an experimental receiver station for satelliteand aircraft-downlinks. It consists of a 4.5m-dome housing a 40-cm steerable mirror telescope on an azimuth/elevation mount and can be configured with various instruments depending on the actual demands of a measurement campaign. An outside view of the station on top of the institute's building is shown in Figure 3 (left). The clamshell dome is located on the roof of the operations room where operations monitoring and support tasks can be executed. In Figure 3, right, the telescope can be seen mounted in its aluminum fork mount.
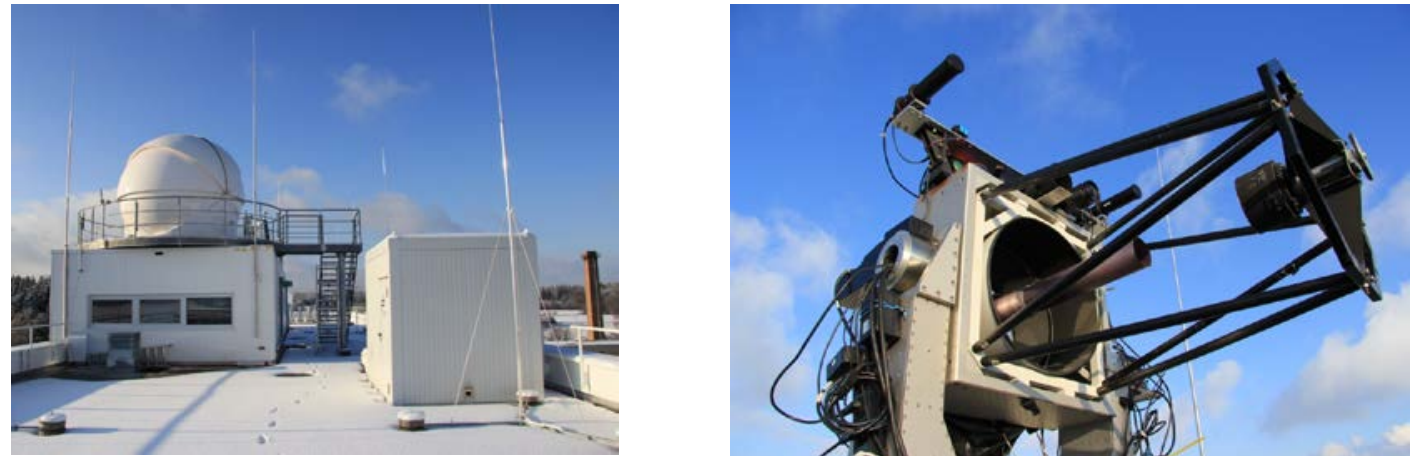

Figure 3. Overview Optical Ground Station Oberpfaffenhofen located on top of the institute building (left) and telescope hold by aluminum fork mount (right). 
The optical bench hosting most of the control and measurement devices is mounted to the back of the telescope. Figure 4 (left) contains a block diagram to illustrate the basic function of the system: the telescope mount points all devices towards the aircraft. The main telescope receives the $1545 \mathrm{~nm}$ signal which is then guided to the fine pointing assembly (FPA) for beam stabilization. With a coupling system, the beam is split and directed to a narrow field of view camera $(\mathrm{NFoV})$ that produces the input signals to the FPA controller. The other receivers are the individual measurement instruments. Adjacent to the telescope, two beacons (laser diode and EDFA) are installed. A Wide Field of View (WFoV) camera and controller are used to steer the mount and two 5-cm lens telescopes are deployed for power measurements. The right picture in Figure 4 shows these instruments installed on top of the frame. In addition, the objective lens of a VIS monitoring camera is also seen which is not included in the block diagram. The clamshell dome and alt-azimuth-mount construction allow the telescope to be pointed in the full 360-degree range.
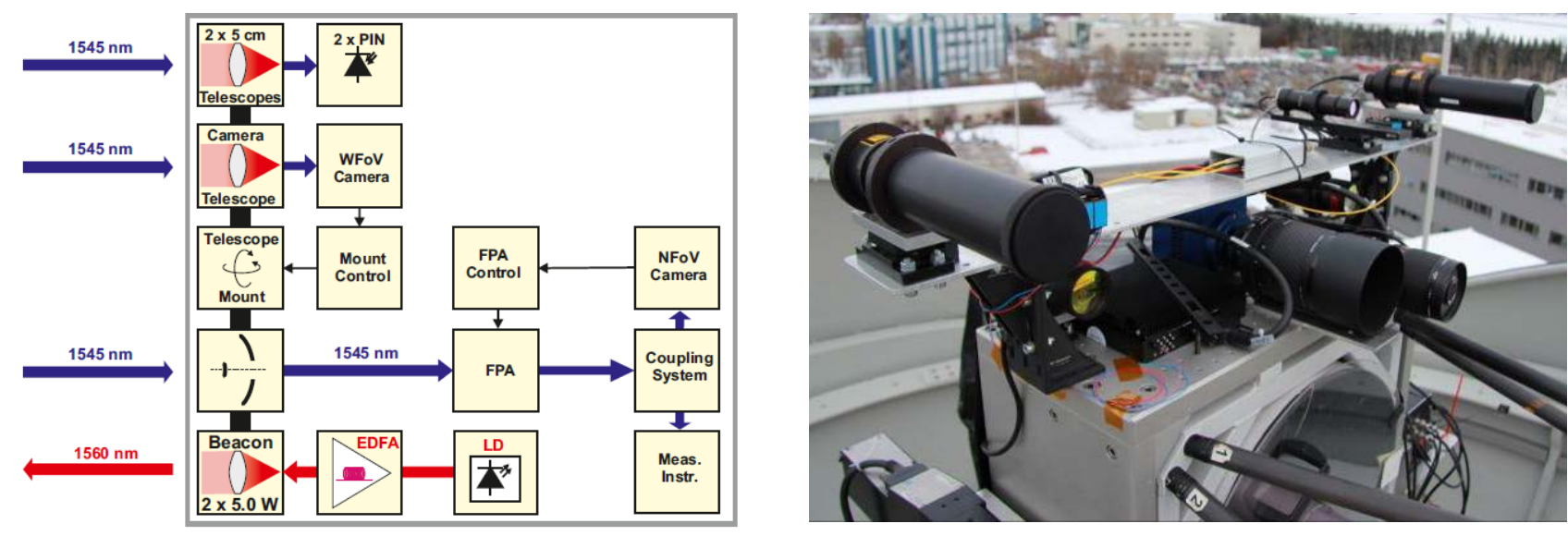

Figure 4. Block diagram of telescope system (left) and close-up of instruments installed in top of telescope frame: NIR coarse field of view camera, VIS monitor camera, beacon telescopes and 5-cm receiver telescopes (with protective cap in place).

\subsection{OGS Optical setup}

A series of beam splitters guide the incoming laser signal to the separate measurement devices. The block diagram in Figure 5 provides a logical sense of how the incoming beam propagates to these devices while Figure 6 shows the overall mechanical setup.

Each measurement camera in the system is connected via a USB connection to a standard desktop PC running Windows where the camera images are stored. However, in the case of the tracking camera, the images are used to provide a feedback signal that corrects pointing errors via the fine-steering mirror.

Complementing the measurements taken with the imaging cameras is a series of PIN diodes that are connected to a data acquisition system. One PIN diode is mounted with the other instruments in the optical path of the 40-cm telescope, effectively measuring the received power across the diameter of the telescope. The other two PIN diodes are connected to 5-cm telescopes and are mounted to the top of the frame of the 40-cm telescope, spaced $1 \mathrm{~m}$ apart (see above).

Crucial to the whole measurement system is the use of a timing signal generator. A square wave timing signal (trigger) with a frequency ranging between $20 \mathrm{~Hz}$ and $80 \mathrm{~Hz}$ is used to synchronize the capture of images in the measurement cameras, thus ensuring that each camera is taking the same snapshot of the atmosphere. The camera control computers are connected to a GPS-based timing system that controls the internal system times of the computers. In essence, the trigger certifies that cameras are correlated in time while the GPS signal certifies that the control computers are correlated in time. When taken together, this method achieves a synchronization within an order of a few milliseconds, the same order of magnitude as typical atmospheric variations, for a much lower cost than more specialized timing systems. 


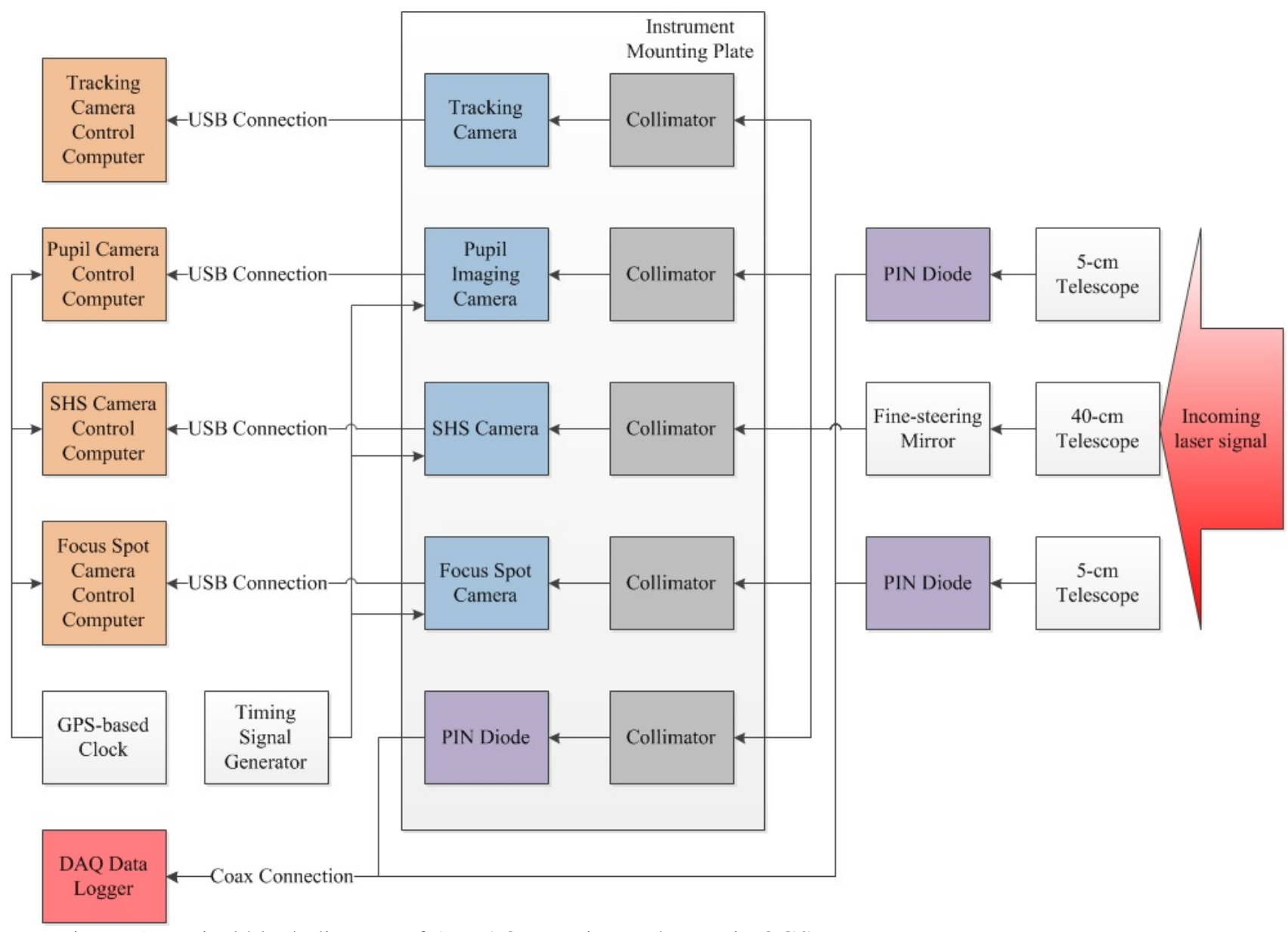

Figure 5. Logical block diagram of ALTAO experimental setup in OGS.

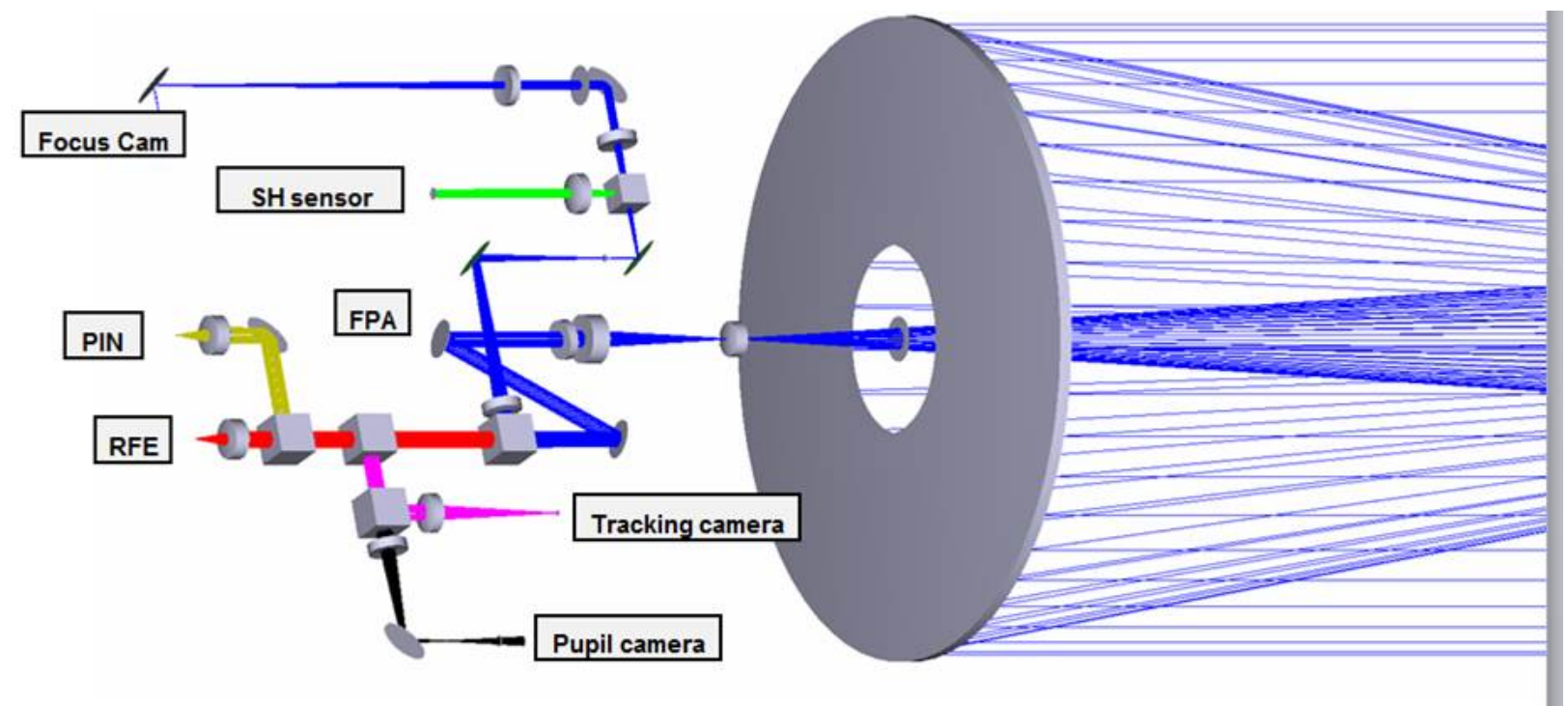

Figure 6. Zemax ray-trace of the optical setup used during the flight campaign. The RFE module was not installed as it was not required for the current flight campaign. 


\subsection{Pupil Plane Camera}

A camera mounted in a pupil plane of the 40-cm telescope was used to measure the intensity distribution of the incoming signal beam across the diameter of the telescope. From images captured with this camera, statistics of the speckle pattern can be derived and a calculation of the atmospheric correlation length (i.e. Fried parameter, $r_{0}$ ) can be made. Table 1 shows the specification of the optical path that provides pupil plane images. The particular camera used here was chosen for its combination of high frame rate, which ensures a temporal sampling sufficient to capture short-term turbulence effects, and moderately sized pixel pitch that provides sufficient spatial resolution to make a reliable calculation of $\mathrm{r}_{0}$.

Table 1. Specifications for the pupil plane camera system.

\begin{tabular}{|c|c|}
\hline Camera manufacturer & Xenics Infrared Solutions \\
\hline CCD dimensions & $320 \times 256$ pixels \\
\hline Pixel pitch & $30 \mu \mathrm{m}$ \\
\hline Spectral response & $0.4-1.7 \mu \mathrm{m}$ \\
\hline Frame rate & Up to $100 \mathrm{~Hz}$ \\
\hline Primary aperture diameter & $40 \mathrm{~cm}$ \\
\hline Pupil size & $3.8 \mathrm{~mm}$ \\
\hline Compression factor & 105 \\
\hline
\end{tabular}

For the purposes of consistency between the different measurements, the same camera type was used for the focal spot measurements and the Shack-Hartmann wavefront sensing. Figure $\mathbf{7}$ shows a sample image taken from one of the data segments collected during flight operations on June $6^{\text {th }}$, 2013.

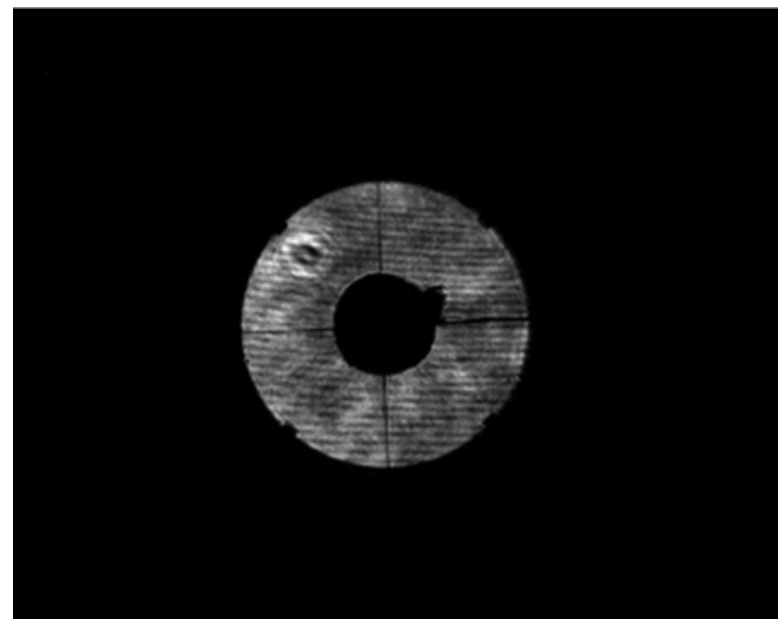

Figure 7. Sample pupil image during flight operations on June 6th, 2013.

\subsection{Focus Spot Camera}

The purpose of camera is to capture an instantaneous image of the focal speckle pattern from which the Fried parameter can be derived. This value for the Fried parameter can then be validated against the value calculated from the other instruments.

The derivation of $r_{0}$ from a focal speckle pattern is important when considering mobile optical links. In such scenarios, as the communications target is being tracked, two important issues become apparent [10]:

- the volume of atmosphere through which the communications laser is propagating is changing rapidly due to the slew rate of the communications terminal and, hence, cannot be considered to be isotropic;

- by virtue of the relative motions between the two terminals, a tracking error is introduced into the system that cannot necessarily be distinguished from the atmospheric angle of arrival 
measurements required for calculating $\mathrm{r}_{0}$ by other means, thus changing the statistics of the $\mathrm{r}_{0}$ calculation.

For these reasons, the focal speckle pattern analysis provides an attractive alternative for determining $r_{0}$ since it requires statistics over shorter measurement periods. Figure 8 shows one of the focal speckle pattern images taken during flight operations on June $6^{\text {th }}$, 2013. Table 2 provides an overview of the specifications for the focus camera system.

Table 2. Specifications for the focus camera system.

\begin{tabular}{|c|c|}
\hline Camera manufacturer & Xenics Infrared Solutions \\
\hline CCD dimensions & $320 \times 256$ pixels \\
\hline Pixel pitch & $30 \mu \mathrm{m}$ \\
\hline Spectral response & $0.4-1.7 \mu \mathrm{m}$ \\
\hline Frame rate & $\mathrm{Up} \mathrm{to} 100 \mathrm{~Hz}$ \\
\hline Primary aperture diameter & $40 \mathrm{~cm}$ \\
\hline System effective focal length & $2400 \mathrm{~mm}$ \\
\hline
\end{tabular}

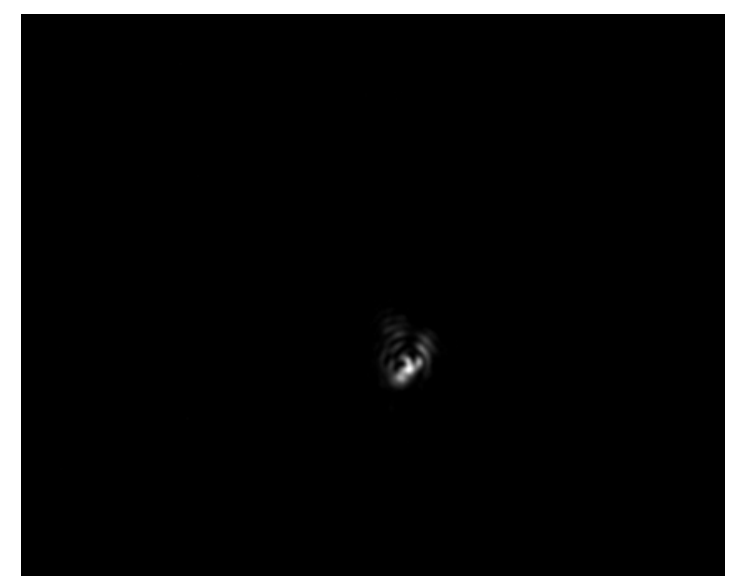

Figure 8. Sample focal spot image taken during flight operations on June 6th, 2013.

\subsection{Shack-Hartmann Wave-front Sensor}

Shack-Hartmann wavefront sensor (SHWS) is designed to estimate the phase distortion of optical fields in real time with an appropriately high fidelity. Shack-Hartmann sensing enables one to combine phase (or tilt) correlation. The principle used to derive a turbulence profile from a SHWS is the same as that used for SCIDAR except that it is the phase instead of the magnitude that is correlated.

The camera used in the Shack-Hartmann optical path is mounted in a pupil plane of the measurement system. A ShackHartmann lenslet array was mounted in a pupil plane in front of the camera's CCD. The position of the camera was then adjusted to provide a focused square grid of spots on the detector. Table 3 lists the specifications of the Shack-Hartmann system used during the flight campaign.

The specification of the lenslet array was chosen such that each focus spot was projected onto a grid of 5 pixels on the camera CCD array, ensuring enough spatial resolution of the spot movement to detect changes in the wavefront without interfering with the detection of the focus spots from adjacent lenslets. Given the compression factor of the 40-cm telescope is 80 , the effective resolution of each lenslet is approximately $1.2 \mathrm{~cm} /$ lenset. 
Table 3. Specifications for Shack-Hartmann wavefront sensing system.

\begin{tabular}{|c|c|}
\hline Camera manufacturer & Xenics Infrared Solutions \\
\hline Lenslet manufacturer & Advanced Micro-Optic Systems \\
\hline Lenslet pitch & $150 \mu \mathrm{m}$ \\
\hline Lenslet radius of curvature & $1.6 \mathrm{~mm}$ \\
\hline Lenslet array size & R $<0.5 \%$ at $1550 \mathrm{~mm}$ \\
\hline AR-coating & UV-grade fused silica \\
\hline Material & $40 \mathrm{~cm}$ \\
\hline Primary aperture diameter & $5.0 \mathrm{~mm}$ \\
\hline Pupil size & 80 \\
\hline Compression factor & \\
\hline
\end{tabular}

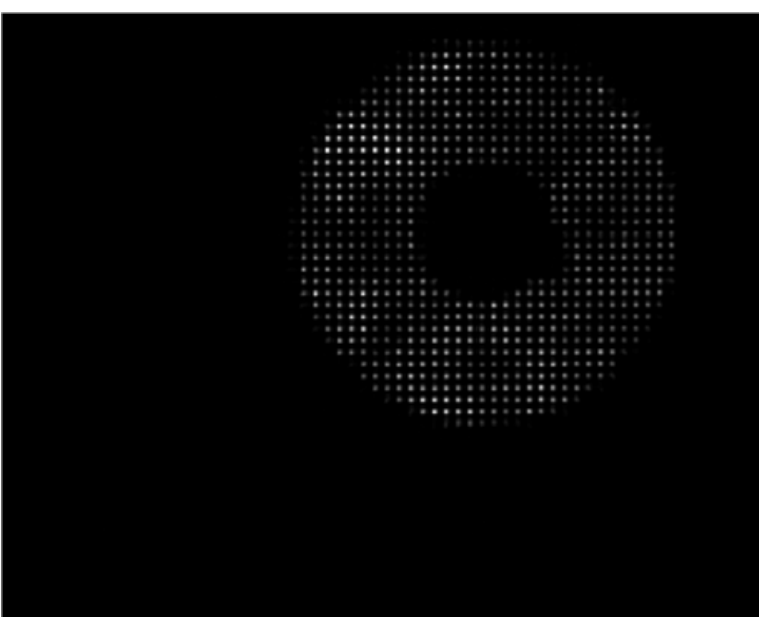

Figure 9. Sample Shack-Hartmann image taken during flight operations on June 6th, 2013.

\subsection{PIN Diodes}

The primary purpose of the PIN diodes is to make power measurements across the diameter of the received communications beam. By measuring the power at various points across the beam diameter, a comparison of scintillation between large and small diameter apertures can be made. Table 4 lists the specifications of the PIN diode system used during the flight campaign. The PIN diodes with the 5-cm aperture were the ones mounted above the 40-cm telescope, separated by $1 \mathrm{~m}$.

Table 4. PIN diode system specification.

\begin{tabular}{|c|c|c|}
\hline Manufacturer & \multicolumn{2}{|c|}{ Thorlabs } \\
\hline Detector size & \multicolumn{2}{|c|}{$1 \mathrm{~mm}$} \\
\hline Spectral response & \multicolumn{2}{|c|}{$0.7-1.8 \mu \mathrm{m}$} \\
\hline Bandwidth & \multicolumn{2}{|c|}{$10 \mathrm{MHz}$} \\
\hline Material & \multicolumn{2}{|c|}{ InGaAs } \\
\hline Aperture & $5 \mathrm{~cm}$ & $40 \mathrm{~cm}$ \\
\hline Effective focal length & $300 \mathrm{~mm}$ & $1200 \mathrm{~mm}$ \\
\hline
\end{tabular}

Each of the three PIN diodes was connected to a National Instruments DAQ system with 16-bit channels and was sampled at a rate of $20 \mathrm{kHz}$. Figure 10 shows a sample of the normalized power received by each of the mounted PIN diodes. 

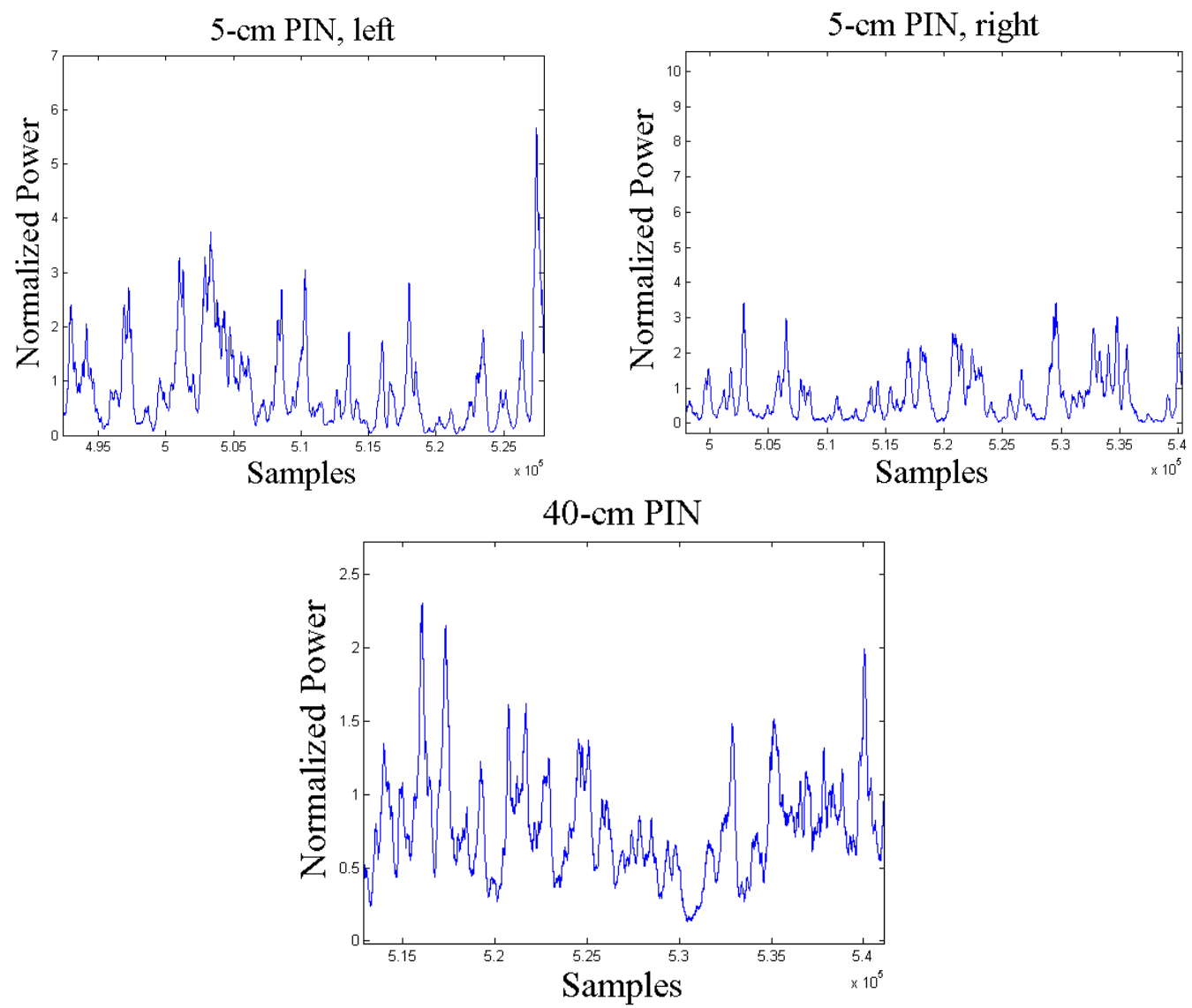

Figure 10. Sample power vectors from the three PIN diodes used during the flight campaign.

\section{FLIGHT CAMPAIGN}

The ALTAO flight campaign consisted of a number of flights during September and October 2012 and the beginning of June 2013. The decision to fly on any given day was primarily dictated by the weather conditions so the operations team maintained a constant state of readiness during each operations period. Table 5 shows the operations timeline for a typical flight day. The personnel involved in the execution of the operations timeline are broken down into two groups; a Flight Operations (Flight Ops) team consisting of the people responsible for aircraft operations (e.g. pilot, co-pilot etc.); an Experiment team consisting of the people responsible for the operation of the optical communication link. The Experiment team is further broken down into an OGS crew who is responsible for the operation of the OGS and a flight terminal operations crew (FTOC), at most two people, who are responsible for the operation of the optical communications terminal on board the aircraft.

This timeline attempts to include enough margin for the various tasks such that it can support both day and night flight operations. The 2013 flight campaign focused on nighttime flights in an effort to mitigate the effect of background radiance since this had been identified as an issue from previous daytime missions.

The flight plan for the day was selected from a list of possible options derived for a previous project involving flight campaigns [11]. The possible flight paths consisted of the following options:

1) Semi-circles ranging in radius from $10 \mathrm{~km}$ to $100 \mathrm{~km}$ with the OGS located at the center

2) Radial paths ranging in distance from $10 \mathrm{~km}$ to $100 \mathrm{~km}$ from the OGS

3) Tangential paths

4) Any combination of the above 
Semi-circular paths were chosen if the experiment team wanted to maintain a constant received radiance at the OGS. However, changing the aircraft's azimuthal position inherently introduces the possibility of measuring different atmospheric conditions over vastly different terrain (i.e. the mountainous terrain of the Alps in the south versus the flatter terrain to the east and west of the OGS). These paths also introduced additional tracking errors as a result of the telescope mount trying to keep up with the angular velocity of the aircraft. Conversely, radial paths were chosen to mitigate the issues related to the semi-circular paths but required constant adjustment of the transmit power on the aircraft as well as adjustments to the attenuation of the measurement devices to prevent them from saturating. Tangential paths were not selected for this campaign. Figure 11 shows the flight path flown in the evening of June $6^{\text {th }}$, 2013 (the red circle indicates the position of the OGS). Consisting of a radial path and several semi-circular paths, this flight plan is an excellent example of the combinations possible from the above options.

Table 5. Operations timeline for a typical flight day.

\begin{tabular}{|c|c|c|}
\hline Operations Time & \begin{tabular}{|l} 
Event \\
\end{tabular} & Duration \\
\hline $\mathrm{T}_{0}-7$ hrs. $0 \mathrm{~min}$. & Weather briefing with Flight Ops team & 0 hrs. 15 min. \\
\hline $\mathrm{T}_{0}-6$ hrs. $30 \mathrm{~min}$ & $\begin{array}{l}\text { Operations planning briefing with } \\
\text { Experiment team }\end{array}$ & 0 hrs. 30 min. \\
\hline $\mathrm{T}_{0}-6$ hrs. 0 min. & $\begin{array}{l}\text { Reconciliation and completion of } \\
\text { identified pre-flight action items }\end{array}$ & 2 hrs. 15 min. \\
\hline $\mathrm{T}_{0}-3$ hrs. $45 \mathrm{~min}$. & Pre-flight briefing & 0 hrs. 15 min. \\
\hline $\mathrm{T}_{0}-2$ hrs. 30 min. & $\begin{array}{c}\text { Activation of OGS systems and } \\
\text { measurement devices }\end{array}$ & 1 hrs. $30 \mathrm{~min}$. \\
\hline $\mathrm{T}_{0}-1$ hr. 0 min. & OGS operation verification with test link & 0 hrs. $20 \mathrm{~min}$. \\
\hline $\mathrm{T}_{0}-0$ hrs. $40 \mathrm{~min}$. & Pre-flight check & 0 hrs. $10 \mathrm{~min}$. \\
\hline $\mathrm{T}_{0}-0$ hrs. $30 \mathrm{~min}$. & Aircraft boarding time & 0 hrs. $15 \mathrm{~min}$. \\
\hline $\mathrm{T}_{0}$ & Take-off & N/A \\
\hline $\mathrm{T}_{0}+0$ hrs. $10 \mathrm{~min}$. & Flight measurements & 2 hrs. 0 min. \\
\hline $\mathrm{T}_{0}+2$ hrs. $10 \mathrm{~min}$. & Return to airport & 0 hrs. $10 \mathrm{~min}$. \\
\hline $\mathrm{T}_{0}+2$ hrs. $20 \mathrm{~min}$. & Landing & N/A \\
\hline $\mathrm{T}_{0}+2$ hrs. $40 \mathrm{~min}$. & Flight debriefing & 0 hrs. $30 \mathrm{~min}$. \\
\hline
\end{tabular}

The initial weather briefing with DLR's Flight Ops team, followed by the Operations Planning Briefing (OPB) with the experiment team, provided the first go/no-go decision opportunity for the day's operations. Given the geography surrounding DLR's OGS, the weather can be quite volatile and the distances covered in the available flight plans can traverse multiple storm cells. In one case, a mission was canceled due to thunderstorm conditions over the Alps to the southeast of the OGS despite clear conditions closer to the OGS.

By the conclusion of the OPB, any issues requiring attention in the experimental setup prior to the flight had been identified at which point the experiment team turns their attention to resolving them. Given the limited time, only issues such as minor software or hardware anomalies could be addressed (e.g. refocusing of measurement cameras). Issues involving a reconfiguration of the optical setup or measurement software upgrades were considered more major and would result in the cancellation of the day's flight. This decision would be taken at the Pre-flight Briefing (PFB), the second go/no-go decision opportunity. A last minute weather update would also be taken into account during the PFB.

Following the PFB, the OGS systems and measurement devices are powered up and the station is readied for flight operations. Once the station had been powered up, the telescope was pointed towards a testlink to perform a final verification, ensuring all systems are in working order. The testlink consists of a $1550 \mathrm{~nm}$ laser source located on a building rooftop approximately $2 \mathrm{~km}$ from the ground station and allows for testing the telescope pointing systems and ensuring proper alignment of the measurement devices (i.e. the beam spot is in focus and centered in the instruments' field of view). The completion of the tests with the testlink marked the final opportunity for the OGS operations crew to make a go/no-go decision for the flight. Only in extreme circumstances could the OGS operations crew recall a "go" decision. 


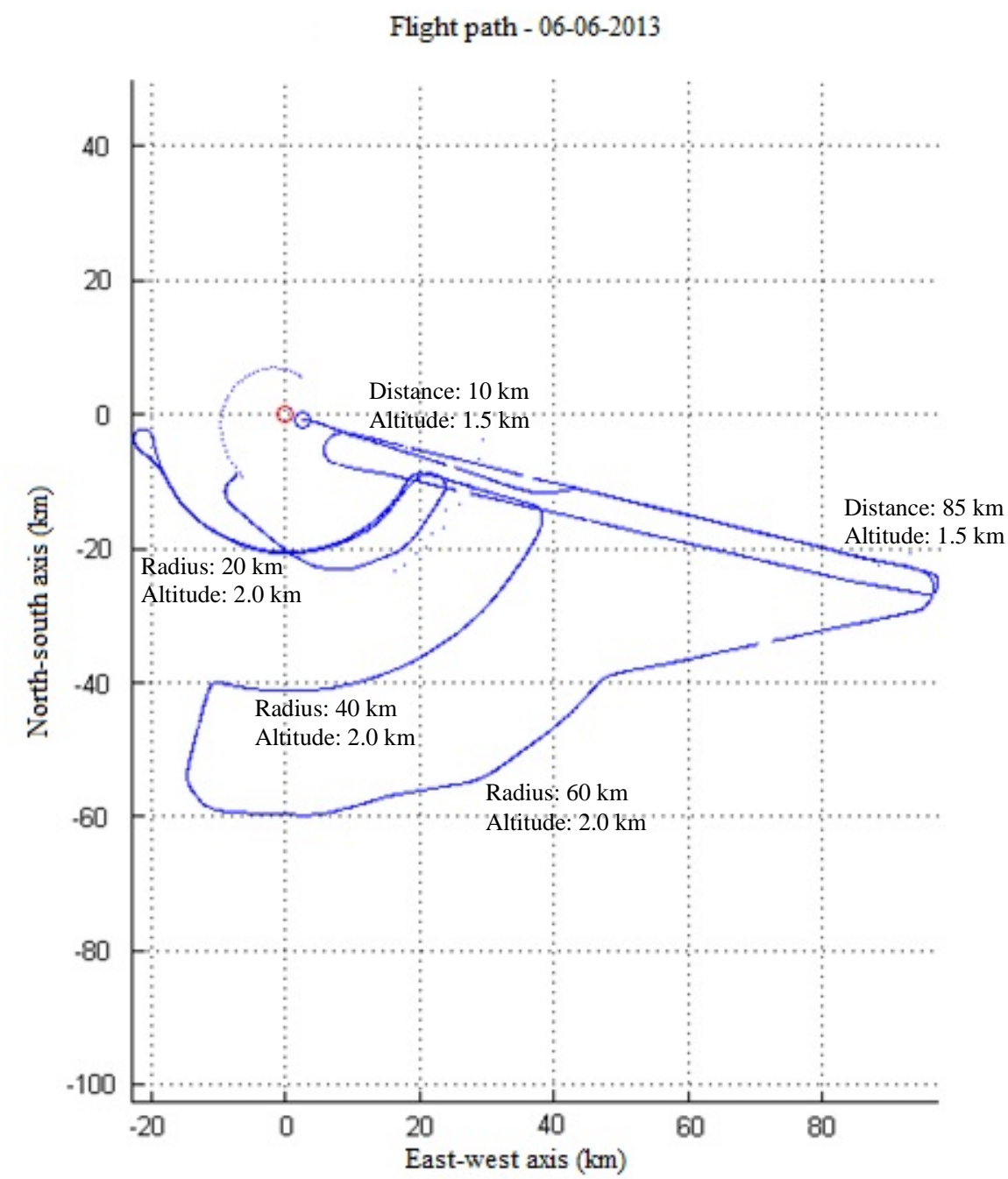

Figure 11. Flight path flown on the evening of June 6th, 2013 between 19:30 and 22:00. Distances are measured in kilometers north-south and east-west relative to the ground station (red circle). Weather conditions for this flight were clear with light winds.

Upon departure from the airport, the aircraft proceeded to the first waypoint of the flight plan. During the transit time, the communications payload was powered up and the acquisition phase of the communications link was initiated. During the 2-hour flight time, measurements were taken using the OGS instrumentation.

At the completion of the flight, the experiment team met for a flight de-briefing to discuss activities during the flight. Anomalies during the flight were discussed and action items assigned, if necessary, in preparation for the next OPB the following day.

In the event that a "no-go" decision is made at any time during the operations timeline, the experiment team would stand down until the OPB the following day.

The following table provides an overview of the successful flights undertaken during the course of the ALTAO project. 
Table 6. Overview of ALTAO flight campaign.

\begin{tabular}{|c|c|c|c|c|c|}
\hline Flight Date & Time of Day & Duration & Weather & Flight path & $\begin{array}{c}\text { Altitude } \\
\text { (above sea level) }\end{array}$ \\
\hline Sept. 28 $8^{\text {th }}, 2012$ & Day & $2.5 \mathrm{hrs}$ & $90 \%$ clear & $\begin{array}{c}21-\mathrm{km} \text { semi- } \\
\text { circles }\end{array}$ & $2 \mathrm{~km}$ \\
\hline Oct. 2 $^{\text {nd }}, 2012$ & Day & $2 \mathrm{hrs}$ & $90 \%$ clear & $\begin{array}{c}20 \mathrm{~km}-\mathrm{semi}- \\
\text { circles }\end{array}$ & $1.5 \mathrm{~km}$ \\
\hline June $^{\text {th }}$, 2013 & Night & $2.5 \mathrm{hrs}$ & Clear & $\begin{array}{c}20-\mathrm{km} \text { semi- } \\
\text { circles; radial } \\
\text { paths }\end{array}$ & $2 \mathrm{~km}, 1.5 \mathrm{~km}$ \\
\hline June 6 & & Clear & & $\begin{array}{c}20-, 40-, 60-\mathrm{km} \\
\text { semi-circles; } \\
\text { radial paths }\end{array}$ & $2 \mathrm{~km}, 1.5 \mathrm{~km}$ \\
\hline
\end{tabular}

\section{DISCUSSION AND CONCLUSION}

The 2013 flight campaign marked the next step in the continuing evolution of DLR's OGS as a platform for supporting optical communications links for a variety of applications. This flight campaign in particular saw a significant improvement in the synchronization of the measurement devices and a greater formalization of the operational procedures necessary for carrying out a successful flight trial. At the same time, new areas of enhancement have been identified for future flight campaigns. Detailed analysis from the data collected during the flight campaign is currently under preparation.

From an operational standpoint, the steps taken to further refine and formalize the operations timeline between the first second flights resulted in a more streamlined procedure, in turn, leading to better quality measurement time. It was noted throughout the flight campaign that there were several obstacles such as trees, airport lighting and lightning rods along the horizontal line of sight that hindered operations. While such obstacles are comparatively small, they often led to losses of the optical link for up to several minutes and significant changes in the aircraft's position. For future flight campaigns, enhancements to the flight plans will help mitigate the impact of these obstacles and will result in more time spent collecting measurement data.

From a data collection standpoint, early indications from the ongoing data analysis show that there are several data segments from each instrument in the OGS that are providing workable results. Since considerable time was invested in improving the synchronization of the OGS instrumentation, the next step in the analysis will be to compare the results between each instrument to confirm their validity. A more quantitative statement can be made about the quality of the data can only upon completion of the comparative analysis.

\section{REFERENCES}

[1] Tolker-Nielsen, T., "In-orbit test result of an operational optical intersatellite link between ARTEMIS and SPOT4, SILEX”, Proc. SPIE 4635, April 30, 2002

[2] "Lunar Laser Communication Demonstration on LADEE achieves world-record data download speeds from the moon”, October 2013, http://www.ll.mit.edu/news/LLCDachievesrecorddatadownloadspeed.html, (December 20, 2013)

[3] Leitgeb, E., Gebhart, M., Fasser, P., Bregenzer, J., Tanczos, J., "Impact of atmospheric effects in free-space optics transmission systems”, Proc. SPIE 4976, April 30, 2003.

[4] Philips, R.L., Andrews, L.C., "Measured statistics of laser-light scattering in atmospheric turbulence”, Journal of the Optical Society of America, Vol. 71, No. 12, p.p. 1440-1445 (1981).

[5] Arimoto, Y., Hayano, Y., "High-speed optical feeder-link system using adaptive optics”, Proc. SPIE 2990, April 24, 1997. 
[6] Mostafa, A., Hranilovic, S., "Channel Measurement and Markov Modeling of an Urban Free-Space Optical Link”, Journal of Optical Communications and Networking, Vol. 4, Issue 10, p.p. 836-846 (2012).

[7] Horwath, J., Fuchs, C., "Aircraft to ground unidirectional laser-comm. terminal for high resolution sensors", Proc. SPIE 7199, (2009).

[8] Schmidt, C., Horwath, J., Shrestha, A., Moll, F., Brechtelsbauer, M., Fuchs, C., "High-speed, high volume optical communication for aircraft”, SPIE Newsroom. SPIE. DOI: 10.1117/2.1201310.005174 (2013).

[9] J. Horwath, C. Fuchs, "Aircraft to Ground Unidirectional Laser-Comm. Terminal for High Resolution Sensors" Proc. SPIE 7199, p. 719909, 2009.

[10] Giggenbach, D., "Deriving an estimate for the Fried parameter in mobile optical transmission scenarios", Applied Optics, Vol. 50, Issue 2, p.p. 222-226 (2011).

[11] Moll, F., Nauerth, S., Fuchs, C., Horwath, J., Rau, M., Weinfurter, “Communication system technology for demonstration of BB84 quantum key distribution in optical aircraft downlinks”, Proc. SPIE 8517, (2012). 